\title{
BERNSTEIN APPROXIMATION PROBLEM FOR DIFFERENTIABLE FUNCTIONS AND QUASI-ANALYTIC WEIGHTS
}

\author{
BY \\ GUIDO ZAPATA $\left.{ }^{1}\right)$
}

\begin{abstract}
The Bernstein problem for differentiable functions is considered. Sufficient conditions in order that a decreasing family of weights be fundamental are given. Some of these conditions are al so related to the concept of quasianalytic weight.
\end{abstract}

1. Introduction. The Bernstein problem for continuous functions is equivalent to finding necessary and sufficient conditions for localizability (see Nachbin [3]). Also, the Bernstein problem has a solution only in a particular case (see Mergelyan [2]). On the other hand, many outstanding results dealing with sufficient conditions for localizability depend on statements about fundamental weights (see Nachbin [3] and Nachbin, Machado and Prolla [4] for results and other references). So it is interesting to give sufficient conditions for a weight to be fundamental. Among those sufficient conditions for localizability, the quasianalytic criterion proved to be of wide use (see [3] and [4]).

In this paper we continue the research on Bernstein problem for differentiable functions. This was initiated in Zapata [6] and was partly announced in Zapata [7]. This problem has also a solution in a particular case (see Sibony [5]). Here we give only sufficient conditions for a family of weights to be fundamental, these conditions being related to the concept of quasi-analytic weight (namely Theorems 1 and 2). Also, the concept of quasi-analytic weight is behind the quasi-analytic criterion of localizability.

The contents of this paper are, for the most part, either results or generalization of results contained in the author's $\mathrm{Ph}$. D. thesis. This was written under the direction of Professor Leopoldo Nachbin, to whom the author expresses his gratitude.

2. Preliminaries. Let $V=\left(V_{a}\right), a \in \mathbf{N}_{m}^{n}$, be a family of directed sets of weights on $\mathbf{R}^{n}$, where $\mathbf{N}_{m}^{n}$ denotes the set of multi-indexes $a=\left(a_{1}, \ldots, a_{n}\right) \in \mathbf{N}^{n}$

Received by the editors November 15, 1972.

AMS (MOS) subject classifications (1970). Primary 41A10; Secondary 46E10.

Key words and phrases. Bemstein problem for differentiable functions, fundamental family of weights, decreasing family, quasi-analytic.

(1) The author was partially supported by Fundo Nacional de Ciencias e Tecnologia (FINEP), Brasil. 
of order $|a|=\alpha_{1}+\cdots+\alpha_{n} \leq m$ and $m \in \mathbf{N} \cup\{+\infty\}, n \in \mathbf{N}^{*}$. The vector space $\mathcal{E}^{m} V_{\infty}\left(\mathbf{R}^{n} ; \mathbf{K}\right)$ of all $m$-times continuously differentiable $\mathbf{K}$-valued functions $f$ on $\mathbf{R}^{n}$ such that $v_{\alpha} \cdot \partial^{\alpha} f$ tends to zero at infinity for every $v_{a} \in V_{a}$ and $a \in \mathbf{N}_{m}^{n}$ will be given the topology $\omega_{v}$ defined by the family of semi-norms

$$
f \mapsto \sup \left\{v_{\alpha}(t)\left|\partial^{\alpha} f(t)\right| ; t \in \mathbf{R}^{n}\right\}
$$

for all such $v_{a}$ and $a$, where $\mathbf{K}$ denotes either $\mathbf{R}$ or $\mathbf{C}$. In particular, when each $V_{a}$ consists of a single weight $v_{a}$ and we consider the family $v=\left(v_{a}\right), a \in \mathbf{N}_{m}^{n}$, we will write $\mathcal{E}^{m} v_{\infty}\left(\mathbf{R}^{n} ; \mathbf{K}\right)$ in place of $\mathcal{E}^{m} V_{\infty}\left(\mathbf{R}^{n} ; \mathbf{K}\right)$ and will denote the corresponding topology by $\omega_{v}$.

The family $v=\left(v_{a}\right), a \in \mathbf{N}_{m}^{n}$, of weights on $\mathbf{R}^{n}$ is said to be rapidly de. creasing at infinity if $\mathcal{E}^{m} \nu_{\infty}\left(\mathbf{R}^{n} ; \mathbf{K}\right)$ contains the algebra $\mathscr{P}\left(\mathbf{R}^{n} ; \mathbf{K}\right)$ of all $\mathbf{K}$ valued polynomials on $\mathbf{R}^{n}$ or equivalently, if each $v_{a}$ is rapidly decreasing at infinity in the sense of Definition 1.24 of Nachbin [3]. The family $v=\left(v_{a}\right)$, $a \in \mathbf{N}_{m}^{n}$, of weights on $\mathbf{R}^{n}$, rapidly decreasing at infinity, is called fundamental when $\mathscr{P}\left(\mathbf{R}^{n} ; \mathbf{K}\right)$ is dense in $\mathcal{E}^{m} v_{\infty}\left(\mathbf{R}^{n} ; \mathbf{K}\right)$. The Bernstein problem consists in asking for necessary and sufficient conditions for a given family $v$ to be fundamental. The Weierstrass theorem on approximation of differentiable functions by polynomials means that every family $v=\left(v_{a}\right), a \in \mathbf{N}_{m}^{n}$, of weights on $\mathbf{R}^{n}$, each of them having compact support, is fundamental.

Remark 1. It can be shown that $\mathcal{E}^{m} v_{\infty}\left(\mathbf{R}^{n} ; \mathbf{K}\right)$ is always the closure of the set of analytic functions that it contains. Also, it can be shown that, if $v=\left(v_{a}\right)$, $a \in \mathbf{N}_{m}^{n}$, is a fundamental family then each $v_{a}$ is a fundamental weight in the classical sense, that is in the sense of Definition 1.24 (loc. cit.), the reciprocal being an open question.

A family $v=\left(v_{a}\right), \alpha \in \mathbf{N}_{m}^{n}$, of weights on $\mathbf{R}^{n}$ will be called a decreasing family if for every $\alpha, \beta \in \mathbf{N}_{m}^{n}$ with $\beta \leq \alpha$, there exists a constant $C_{a, \beta}$ such that $v_{a} \leq C_{a, \beta} \cdot v_{\beta}$

In the following, we will write $\mathcal{G}^{m} v_{\infty}\left(\mathbf{R}^{n}\right)$ and $\mathcal{P}\left(\mathbf{R}^{n}\right)$ instead of $\mathcal{G}^{m} v_{\infty}\left(\mathbf{R}^{n} ; \mathbf{K}\right)$ and $\mathcal{P}\left(\mathbf{R}^{n} ; \mathbf{K}\right)$, respectively. Also, we will write $\mathfrak{D}^{m}\left(\mathbf{R}^{n} ; \mathbf{K}\right)$ or $\mathfrak{D}^{m}\left(\mathbf{R}^{n}\right)$ for the algebra of all $m$-times continuously differentiable $K$-valued functions on $\mathbf{R}^{n}$ having compact support. We put $\mathfrak{D}\left(\mathbf{R}^{n}\right)=\bigcap_{m \in \mathbb{N}} \mathfrak{D}^{m}\left(\mathbf{R}^{n}\right)$.

\section{Some basic lemmas.}

Lemma 1. Let $v=\left(v_{\alpha}\right)$, $a \in \mathbf{N}_{m}^{n}$, be a decreasing family of weights on $\mathbf{R}^{n}$. Then $\mathfrak{D}^{m}\left(\mathbf{R}^{n}\right)$ is dense in $\mathcal{E}^{m} v_{\infty}\left(\mathbf{R}^{n}\right)$.

Proof. Let $f \in \mathcal{E}^{m} v_{\infty}\left(\mathbf{R}^{n}\right), m^{\prime} \in \mathbf{N}, m^{\prime} \leq m$, and $\epsilon>0$ be given. Since the set $\left\{t\left|v_{a}(t)\right| \partial^{a} f(t) \mid \geq \epsilon / 2\right\}$ is compact, for every $\alpha$, and since $N_{m}^{n}$ is finite, there exists a compact $K$ such that $t \notin K$ implies $v_{a}(t)\left|\partial^{a} f(t)\right|<\epsilon / 2$ for all $a \in \mathbf{N}_{m}^{n}$. 
Let $\lambda>0$ be given. There exists $\theta_{\lambda} \in \mathscr{D}^{m}\left(\mathbf{R}^{n}\right)$, real, such that $0 \leq \theta_{\lambda} \leq 1$, $\theta_{\lambda} \mid K=1$ and $\sup \left\{\left|\partial^{\beta} \theta_{\lambda}(t)\right| ; t \in \mathbf{R}^{n}\right\} \leq \lambda$ for all $\beta \in \mathbf{N}_{m}^{n}$, such that $\beta \neq 0$. Let $\alpha \in \mathbf{N}_{m}^{n}$, and $t \in \mathbf{R}^{n}$ be given. If $\alpha=0$ then $v_{0}(t)\left|f(t)-\left(\theta_{\lambda} \cdot f\right)(t)\right|=$ $v_{0}(t)|f(t)|\left|1-\theta_{\lambda}(t)\right|$. So

$$
v_{0}(t)\left|f(t)-\left(\theta_{\lambda} \cdot f\right)(t)\right|<\epsilon / 2
$$

If $a \neq 0$, then

$$
\begin{aligned}
& v_{\alpha}(t)\left|\partial^{\alpha} f(t)-\partial^{\alpha}\left(\theta_{\lambda} \cdot f\right)(t)\right| \\
& \quad \leq v_{\alpha}(t)\left|\partial^{\alpha} f(t)\right|\left|1-\theta_{\lambda}(t)\right|+\sum_{\beta \leq \alpha, \beta \neq 0}\left(\begin{array}{l}
\alpha \\
\beta
\end{array}\right)\left|\partial^{\beta} \theta_{\lambda}(t)\right| v_{a}(t)\left|\partial^{\alpha-\beta} f(t)\right| .
\end{aligned}
$$

Since $v_{a} \leq C_{a, a-\beta} \cdot v_{\beta}$ and $\beta \neq 0$ we have

$$
\begin{aligned}
\left|\partial^{\beta} \theta_{\lambda}(t)\right| v_{a}(t)\left|\partial^{\alpha-\beta} f(t)\right| & \\
& \leq \lambda C_{a, a_{-} \beta} \sup \left\{v_{a_{-} \beta}(s)\left|\partial^{\alpha_{-} \beta} f(s)\right| ; s \in \mathbf{R}^{n}\right\} .
\end{aligned}
$$

So, we can take $\lambda$ small enough such that

$$
\sum_{\beta \leq a, \beta \neq 0}\left(\begin{array}{l}
a \\
\beta
\end{array}\right)\left|\partial^{\beta} \theta_{\lambda}(t)\right| v_{a}(t)\left|\partial^{a-\beta} f(t)\right|<\frac{\epsilon}{2} .
$$

Since $v_{a}(t)\left|\partial^{a} f(t)\right|\left|1-\theta_{\lambda}(t)\right|<\epsilon / 2$ we get

$$
v_{a}(t)\left|\partial^{a} f(t)-\partial^{a}\left(\theta_{\lambda} \cdot f\right)(t)\right|<\epsilon .
$$

Letting $\phi=\theta_{\lambda} \cdot f$ we have $\phi \in \mathfrak{D}^{m}\left(\mathbf{R}^{n}\right)$ and from (1) and (2) we get

$$
\sup \left\{v_{a}(t)\left|\partial^{\alpha} f(t)-\partial^{\alpha} \phi(t)\right| ; t \in \mathbf{R}^{n}\right\}<\epsilon \text { for all } \alpha \in \mathbf{N}_{m^{\prime}}^{n} .
$$

Lemma 2. Let $\Gamma$ be a nonempty set of fundamental weights on $\mathbf{R}$ in the classical sense of Bernstein. Assume that for every $u \in \Gamma$ there exists $u^{\prime} \in \Gamma$ sucb that

(1) $(1+|t|) \cdot u(t) \leq u^{\prime}(t)$, for all $t \in \mathbf{R}$;

(2) $u^{\prime}\left(t_{2}\right) \leq u^{\prime}\left(t_{1}\right)$ for all $t_{1}, t_{2} \in \mathbf{R},\left|t_{1}\right| \leq\left|t_{2}\right|$. Let $v=\left(v_{i}\right), 0 \leq i \leq m$, be a decreasing family of weights on $\mathbf{R}$ such that $v_{0} \leq u$ for some $u \in \Gamma$. Then $v$ is fundamental.

Proof. It is enough to assume $m$ finite and $K=\mathbf{R}$. We will use induction on $m$. In fact, when $m=0$ the conclusion is trivially verified. Letting $m$ be an integer $\geq 1$, assume the lemma to be true for $m-1$. From the hypothesis on $\Gamma$ there exists $u^{\prime} \in \Gamma$ such that (1) and (2) are true. Let $w$ be the family of weights given by

(3) $w_{0}(t)=u^{\prime}(t) /(1+|t|)(t \in \mathbf{R}), w_{\mathrm{i}}=u^{\prime}$ for $i \neq 0$.

We claim that $w$ is fundamental. In fact, let $f \in \mathcal{E}^{m} w_{\infty}(\mathbf{R})$ and $\epsilon>0$ be 
given. If $v^{\prime}$ is the family of weights given by $v_{j}^{\prime}=u^{\prime}$ for all $j, 0 \leq j \leq m-1$, then $v^{\prime}$ is fundamental by the induction hypothesis. Since $w_{j+1}=v_{j}^{\prime}$ for $0 \leq j \leq$ $m-1$ we have $f^{\prime} \in \mathcal{E}^{m-1} \nu_{\infty}^{\prime}(\mathbf{R})$ so there exists $P_{0} \in \mathcal{P}(\mathbf{R})$ such that

(4) $\sup \left\{u^{\prime}(s)\left|\left(f^{\prime}\right)^{(j)}(s)-P_{0}^{(j)}(s)\right| ; s \in \mathbf{R}\right\}<\epsilon$ for $0 \leq j \leq m-1$.

Let $P \in \mathscr{P}(\mathbf{R})$ be such that

(5) $P^{\prime}=P_{0}, P(0)=f(0)$.

Let $t \in \mathbf{R}$ be given. The mean value theorem and (5) give $|f(t)-P(t)|=$ $|t|\left|f^{\prime}(s)-P_{0}(s)\right|$ for some $s$ such that $|s| \leq|t|$. Then from (3) and (2), we get $w_{0}(t)|f(t)-P(t)| \leq u^{\prime}(s)\left|f^{\prime}(s)-P_{0}(s)\right|$. Whence, from (4) it follows that

(6) $w_{0}(t)|f(t)-P(t)|<\epsilon$.

Also, from (5) and (4), it follows that

(7) $w_{i}(t)\left|f^{(i)}(t)-P^{(i)}(t)\right|<\epsilon, 1 \leq i<m$.

Since $t$ is arbitrary, it follows from (6) and (7) that $w$ is fundamental. Notice that, for every $i, 0 \leq i \leq m$, we have $v_{i} \leq$ (constant) $\cdot w_{i}$ so the inclusion $\mathcal{E}^{m} w_{\infty}(\mathbf{R}) \subset \mathcal{E}^{m} v_{\infty}(\mathbf{R})$ is defined and continuous. From this it follows that $\mathcal{P}(\mathbf{R})$ is $\omega_{v}$-dense in $\mathfrak{D}^{m}(\mathbf{R})$. From Lemma $1, \mathfrak{D}^{m}(\mathbf{R})$ is dense in $\mathcal{E}^{m} v_{\infty}(\mathbf{R})$, hence $\mathscr{P}(\mathbf{R})$ is dense in $\mathcal{E}^{m} v_{\infty}(\mathbf{R})$.

If $g_{1}, \cdots, g_{n}$ are $\mathbf{K}$-valued functions defined on $\mathbf{R}$, we denote by $g_{1} \times \cdots \times g_{n}$ the function

$$
\left(t_{1}, \cdots, t_{n}\right) \in \mathbf{R}^{n} \rightarrow g_{1}\left(t_{1}\right) \cdot \ldots \cdot g_{n}\left(t_{n}\right) .
$$

Lemma 3. Let $\Gamma$ be as in Lemma 2. Let $v=\left(v_{a}\right), \alpha \in \mathbf{N}_{m}^{n}$, be a decreasing family of weights on $\mathbf{R}^{n}$ such that there exist $u_{1}, \cdots, u_{n} \in \Gamma$ verifying $v_{0} \leq$ $u_{1} \times \cdots \times u_{n} \cdot$ Then $v$ is fundamental.

Proof. Let $w$ be the family of weights on $\mathbf{R}^{n}$ given by $w_{a}=u_{1} \times \cdots \times u_{n}$, for all $a \in \mathbf{N}_{m}^{n}$. It is clear that $w$ is rapidly decreasing at infinity. We claim that $\mathscr{P}\left(\mathbf{R}^{n}\right)$ is $\omega_{w}$-dense in $\mathfrak{D}^{m}\left(\mathbf{R}^{n}\right)$. Moreover, $w$ is fundamental by Lemma 1 . For $j=1, \cdots, n$ let $w^{j}$ be the family of weights on $\mathbf{R}$ given by $w_{i}^{j}=u_{j}$ for all $i$, $0 \leq i \leq m$. Letting $f_{j} \in \mathcal{E}^{m}\left(w^{j}\right)_{\infty}(\mathbf{R}), j=1, \cdots, n$, then $f_{1} \times \cdots \times f_{n}$ is in $\mathcal{E}^{m} w_{\infty}\left(\mathbf{R}^{n}\right)$. In fact, for every $a=\left(a_{1}, \cdots, a_{n}\right) \in \mathbf{N}_{m}^{n}$ we have $w_{a} \cdot \partial^{a}\left(f_{1} \times \cdots \times f_{n}\right)=$ $\left(u_{1} \cdot \partial^{a_{1}} f_{1}\right) \times \cdots \times\left(u_{n} \cdot \partial^{a_{n}} f_{n}\right)$. Hence $w_{a} \cdot \partial^{a}\left(f_{1} \times \cdots \times f_{n}\right)$ tends to zero at infinity. Also, it follows that the mapping $\pi$ from $\mathcal{G}^{m}\left(w^{1}\right)_{\infty}(\mathbf{R}) \times \cdots \times \mathcal{E}^{m}\left(w^{n}\right)_{\infty}(\mathbf{R})$ into $\mathscr{E}^{m} w_{\infty}\left(\mathbf{R}^{n}\right)$, defined by $\pi\left(f_{1}, \cdots, f_{n}\right)=f_{1} \times \cdots \times f_{n}$, is $n$-linear and continuous. Since $w^{j}(j=1, \cdots, n)$ is fundamental by Lemma 2 , it follows that $\mathscr{D}(\mathbf{R}) \times \cdots \times \mathscr{D}(\mathbf{R})$ ( $n$-times) is contained in the closure of $\mathscr{P}(\mathbf{R}) \times \cdots \times \mathscr{P}(\mathbf{R})$ in the product topology of $\mathcal{E}^{m}\left(w^{1}\right)_{\infty}(\mathbf{R}) \times \cdots \times \mathcal{E}^{m}\left(w^{n}\right)_{\infty}(\mathbf{R})$. By continuity of $\pi$ it follows that $\pi[\mathcal{P}(\mathbf{R}) \times \cdots \times \mathfrak{P}(\mathbf{R})]$ is $\omega_{w}$-dense in $\pi[\mathfrak{D}(\mathbf{R}) \times \cdots \times \mathfrak{D}(\mathbf{R})]$. Let $\mathscr{D}(\mathbf{R}) \otimes \cdots \otimes \mathscr{D}(\mathbf{R})$ denote the vector space generated by $\pi[\mathscr{D}(\mathbf{R}) \times \cdots \times \mathscr{D}(\mathbf{R})]$. 
Since $\pi[\mathscr{P}(\mathbf{R}) \times \cdots \times \mathscr{P}(\mathbf{R})] \subset \mathcal{P}\left(\mathbf{R}^{n}\right)$ and since the closure of $\mathscr{P}\left(\mathbf{R}^{n}\right)$ is also a vector subspace of $\mathcal{E}^{m} w_{\infty}\left(\mathbf{R}^{n}\right)$ we conclude that $\mathcal{P}\left(\mathbf{R}^{n}\right)$ is $\omega_{w}$-dense in $\mathscr{D}(\mathbf{R}) \otimes \cdots \otimes D(R)$. Let $a \in \mathbf{N}_{m}^{n}$ be given. Since $w_{a}$ is bounded, there exists a constant $C>0$ such that

$$
\sup \left\{w_{a}(t)\left|\partial^{\alpha} f(t)\right| ; t \in \mathbf{R}^{n}\right\} \leq C \sup \left\{\left|\partial^{\alpha} f(t)\right| ; t \in \mathbf{R}^{n}\right\}
$$

for every $f \in \mathscr{D}^{m}\left(\mathbf{R}^{n}\right)$ so that the topology induced by $\mathcal{E}^{m} w_{\infty}\left(\mathbf{R}^{n}\right)$ on $\mathfrak{D}^{m}\left(\mathbf{R}^{n}\right)$ is coarser than the topology of uniform convergence of order $m$. From Proposition $1.4, \$ 8$ of Horvath [1], it follows that $\mathscr{D}(\mathbf{R}) \otimes \cdots \otimes \mathscr{D}(\mathbf{R})$ is dense in $\mathfrak{D}^{m}\left(\mathbf{R}^{n}\right)$ in the inductive limit topology, which is finer than the topology of uniform convergence of order $m$. So it follows that $\mathscr{D}(\mathbf{R}) \otimes \cdots \otimes \mathscr{D}(\mathbf{R})$ is $\omega_{w}$-dense in $\mathfrak{D}^{m}\left(\mathbf{R}^{n}\right)$. Hence $\mathscr{P}\left(\mathbf{R}^{n}\right)$ is $\omega_{w}$-dense in $\mathfrak{D}^{m}\left(\mathbf{R}^{n}\right)$, and our claim is proved. To finish the proof, notice that for every $a \in \mathbf{N}_{m}^{n}$ we have $v_{a} \leq$ (constant) $\cdot w_{a}$. So the inclusion $\mathcal{E}^{m} w_{\infty}\left(\mathbf{R}^{n}\right) \subset \mathcal{E}^{m} v_{\infty}\left(\mathbf{R}^{n}\right)$ is defined and continuous. In particular, $v$ is rapidly decreasing at infinity and $\mathcal{P}\left(\mathbf{R}^{n}\right)$ is $\omega_{\nu}$-dense in $\mathfrak{D}^{m}\left(\mathbf{R}^{n}\right)$. From Lemma 1 it follows that $\mathscr{D}^{m}\left(\mathbf{R}^{n}\right)$ is dense in $\mathcal{E}^{m} v_{\infty}\left(\mathbf{R}^{n}\right)$ and we conclude that $v$ is fundamental.

4. Sufficient conditions for a family to be fundamental. Let $u$ be a weight on R. For $k=0,1, \cdots$, put

$$
M(u)_{k}=\sup \left\{u(t)|t|^{k} ; t \in \mathbf{R}\right\}
$$

Assume $M(u)_{k}$ to be finite for all $k$. If

$$
\mu(u)_{k}=\inf \left\{\left(M(u)_{j}\right)^{1 / j} ; j \geq k\right\}
$$

then it is easy to prove the following:

$$
\sum_{k \geq 1}\left[\mu(u)_{k}\right]^{-1}=+\infty \text { if and only if } \sum_{k \geq 1}\left[M(u)_{k}\right]^{-1 / k}=+\infty .
$$

Because of this, a weight that verifies one of these conditions is called quasi-analytic.

Remark 2. Every sequence $\left(M_{k}\right), k \in \mathbf{N}$, of positive numbers that verifies the Denjoy-Carleman condition that is $\Sigma_{k \geq 1}\left(\mu_{k}\right)^{-1}=+\infty$, where $\mu_{k}=$ $\inf \left\{\left(M_{j}\right)^{1 / j} ; j \geq k\right\}$, gives rise to a quasi-analytic weight through

$$
u(t)=\inf \left\{M_{k} /|t|^{k} ; k=0,1, \ldots\right\}, \quad t \in \mathbf{R}, t \neq 0,
$$

and $u(0)=0$ in case some $M_{k}=0, u(0)=M_{0}$ otherwise.

Let $u$ be a weight on $\mathbf{R}^{n}$. Let us consider the following property:

(*) There exist quasi-analytic weights $u_{1}, \cdots, u_{n}$ on $\mathbf{R}$ such that $u \leq u_{1} \times \cdots \times u_{n}$.

Remark 3. Let $u$ be a weight on $\mathbf{R}^{n}$. Assume that $u$ is rapidly decreasing at infinity, that is, for all $\gamma \in \mathbf{N}^{n}$ the function $t \longmapsto u(t)|t|^{\gamma}$ vanishes at infinity, where $|t|^{\gamma}=\left|t_{1}\right|^{\gamma_{1}} \ldots\left|t_{n}\right|^{\gamma_{n}}$ and $0^{0}=1$ by courtesy. Define

$$
\tilde{u}(t)=\inf \left\{M(u)_{\gamma} /|t|^{\gamma} ; \gamma \in \mathbf{N}^{n}\right\}
$$


for all $t \in \mathbf{R}^{n}$, where $M(u)_{\gamma}=\sup \left\{u(s)|s|^{\gamma} ; s \in \mathbf{R}^{n}\right\}$. Then $u$ satisfies property (*) if and only if the restriction of $\tilde{u}$ to each coordinate axis is a quasi-analytic weight. It can be shown that this is no longer true if we just ask the latter condition on $u$ instead of $\tilde{u}$.

Theorem 1. Let $v=\left(v_{a}\right), a \in \mathbf{N}_{m^{\prime}}^{n}$ be a decreasing family of weights on $\mathbf{R}^{n}$ such that $v_{0}$ satisfies property $(*)$. Then $v$ is fundamental.

Proof. Let $u$ be a quasi-analytic weight on R. From Lemma 2.29 of Nachbin [3] it follows that $u$ is fundamental. Also, from the proof of that lemma it follows that the weight $C u^{c}$ is quasi-analytic for all $C, c \in \mathbf{R}$ such that $C \geq 0, c>0$. Define $\tilde{u}$ as in Remark 3. Then $\tilde{u}$ is a quasi-analytic weight. Also $u \leq \tilde{u}$ and $\tilde{u}\left(t_{2}\right) \leq \tilde{u}\left(t_{1}\right)$ for all $t_{1}, t_{2} \in \mathbf{R}$ such that $\left|t_{1}\right| \leq\left|t_{2}\right| \cdot$ Let $u^{\prime}=C \tilde{u}^{1 / 2}$ where $C=\sup \left\{(1+|s|) \tilde{u}^{1 / 2}(s) ; s \in \mathbf{R}\right\}$. Then $u^{\prime}$ is a quasi-analytic weight such that

(1) $(1+|t|) u(t) \leq u^{\prime}(t)$ for all $t \in \mathbf{R}$.

Also

(2) $u^{\prime}\left(t_{2}\right) \leq u^{\prime}\left(t_{1}\right)$ for all $t_{1}, t_{2} \in \mathbf{R}$ such that $\left|t_{1}\right| \leq\left|t_{2}\right|$.

Hence the set of quasi-analytic weights on $\mathbf{R}$ verifies the hypotheses of the set $\Gamma$ as in Lemma 2. From Lemma 3 it follows that $v$ is fundamental.

Theorem 2. Let $v=\left(v_{a}\right), a \in \mathbf{N}_{m}^{n}$, be a decreasing family of weights on $\mathbf{R}^{n}$ sucb that

$$
\sum_{k \geq 1}\left[\bar{M}\left(v_{0}\right)_{k}\right]^{-1 / k}=+\infty
$$

where

$$
\bar{M}\left(v_{0}\right)_{k}=\sum_{\gamma \in \mathbf{N}^{n},|\gamma|=k} \sup \left\{v_{0}(t)|t|^{\gamma} ; t \in \mathbf{R}^{n}\right\} .
$$

Then $v$ is fundamental.

Proof. Let $\left\|\left(t_{1}, \cdots, t_{n}\right)\right\|=\max \left(\left|t_{1}\right|, \cdots,\left|t_{n}\right|\right)$ and let $N_{k}=$ $\sup \left\{v_{0}(t)\left\|_{t}\right\|^{k} ; t \in \mathbf{R}^{n}\right\}, k=0,1, \ldots$ Then $N_{k} \leq \bar{M}\left(v_{0}\right)_{k}$ for all $k$. Define, for each $s \in \mathbf{R}$,

$$
u(s)=\inf \left\{N_{k} /|s|^{k} ; k=0,1, \ldots\right\} .
$$

Then $u$ is a quasi-analytic weight and $v_{0}(t) \leq u(\|t\|), t \in \mathbf{R}^{n}$. This inequality proves that $v$ is rapidly decreasing at infinity. Also, $u\left(s_{2}\right) \leq u\left(s_{1}\right)$ for all $s_{1}$, $s_{2} \in \mathbf{R}$, such that $\left|s_{1}\right| \leq\left|s_{2}\right|$. Let $t=\left(t_{1}, \cdots, t_{n}\right) \in \mathbf{R}^{n}$ be given. Since $\left|t_{j}\right| \leq$ $\|t\|, j=1, \cdots, n$, it follows that $u(\|t\|) \leq u\left(t_{j}\right)$, for all $j$. So, $v_{0} \leq u^{1 / n} \times \cdots$ $\times u^{1 / n}$. Since $u$ is a quasi-analytic weight, $u^{1 / n}$ is too. Hence, $v$ is fundamental by Theorem 1 . 
Corollary. Let $v=\left(v_{a}\right), a \in \mathbf{N}_{m}^{n}$, be a decreasing family of weights on $\mathbf{R}^{n}$. Assume that there exist $c>0$ and $p \in \mathbf{N}$ such that

$$
\sup \left\{v_{0}(t)|t|^{\gamma} ; t \in \mathbf{R}^{n}\right\} \leq\left(c \log _{0}|\gamma| \cdots \log _{p}|\gamma|\right)|\gamma|
$$

for all $\gamma \in \mathbf{N}^{n}$ such that $|\gamma|$ is large enough, where as usual $\log _{0} k=k$ and $\log _{p} k=\log \left(\log _{p-1} k\right)$ if $p \geq 1$. Then $v$ is fundamental.

Proof. In the notation of Theorem 2 we have, for $k \in \mathbf{N}$ large enough,

$$
\bar{M}\left(v_{0}\right)_{k} \leq(k+1)^{n-1}\left(c \log _{0} k \cdots \log _{p} k\right)^{k},
$$

hence the series $\dot{\Sigma}_{k \geq 1}\left[\bar{M}\left(v_{0}\right)_{k}\right]^{-1 / k}$ diverges. The conclusion follows from that theorem.

Remark 4. An important problem that remains unsolved is the following:

Let a family $v=\left(v_{a}\right), a \in \mathbf{N}_{m}^{n}$, of weights on $\mathbf{R}^{n}$ be given such that each $v_{a}$ satisfies property $(*)$. Is it then true that $v$ is fundamental?

\section{BIBLIOGRAPHY}

1. J. Horváth, Topological vector spaces and distributions. Vol. 1, Addi son-Wesley, Reading, Mass., 1966. MR 34 \#4863.

2. S. N. Mergeljan, Weighted approximations by polynomials, Uspehi Mat. Nauk 11 (1956), no. 5 (71), 107-152; English transl., Amer. Math. Soc. Transl. (2) 10 (1958), 59106. MR 18, 734; MR $20 \# 1146$.

3. L. Nachbin, Elements of approximation theory, Van Nostrand, Princeton, N. J., 1967. MR $36 \# 572$.

4. L. Nachbin, S. Machado and J. Prolla, Weighted approximation, vector fibrations and algebras of operators, J. Math. Pures Appl. 50 (1971), 299-323. MR 44 \#3065.

5. N. Sibony, Problème de Bernstein pour les fonctions différentiables, Séminaire Choquet, 1969/70, Initiation à l'analyse, fasc. 2, Exposé 12, Secrétariat mathématique, Paris, 1970. MR 43 \#6632.

6. G. Zapata, Aproximação ponderada para funçōes diferenciáveis, Monografias do Centro Brasileiro de Pesquisas Físicas, vol. 30, Rio de Janeiro, 1971.

7. - Sur le problème de Bernstein et les algèbres de fonctions continûment différentiables, C. R. Acad. Sci. Paris 274 (1972), 70-72.

INSTITUTO DE MATHEMÁTICA, UNIVERSIDADE FEDERAL DO RIO DE JANEIRO, CAIXA POSTAL 1835, ZC-00, 20000 RIO DE JANEIRO, GB, BRASIL 